\title{
Identification of transcriptome and fluralaner responsive genes in the common cutworm Spodoptera litura Fabricius, based on RNA-seq
}

Zhong-Qiang Jia ${ }^{1}$, Di Liu' ${ }^{1}$, Ying-Chuan Peng ${ }^{1,3}$, Zhao-Jun Han ${ }^{1}$, Chun-Qing Zhao ${ }^{1 *}$ and Tao Tang ${ }^{2 *}$

\begin{abstract}
Background: Fluralaner is a novel isoxazoline insecticide with a unique action site on the $\gamma$-aminobutyric acid receptor (GABAR), shows excellent activity on agricultural pests including the common cutworm Spodoptera litura, and significantly influences the development and fecundity of $S$. litura at either lethal or sublethal doses. Herein, Illumina HiSeq Xten (IHX) platform was used to explore the transcriptome of S. litura and to identify genes responding to fluralaner exposure.
\end{abstract}

Results: A total of 16,572 genes, including 451 newly identified genes, were observed in the S. litura transcriptome and annotated according to the COG, GO, KEGG and NR databases. These genes included 156 detoxification enzyme genes [107 cytochrome P450 enzymes (P450s), 30 glutathione S-transferases (GSTs) and 19 carboxylesterases (CarEs)] and 24 insecticide-targeted genes [5 ionotropic GABARs, 1 glutamate-gated chloride channel (GluCl), 2 voltage-gated sodium channels (VGSCs), 13 nicotinic acetylcholine receptors (nAChRs), 2 acetylcholinesterases (AChEs) and 1 ryanodine receptor (RyR)]. There were 3275 and 2491 differentially expressed genes (DEGs) in S. litura treated with $\mathrm{LC}_{30}$ or $L C_{50}$ concentrations of fluralaner, respectively. Among the DEGs, 20 related to detoxification [16 P450s, 1 GST and 3 CarEs] and 5 were growth-related genes (1 chitin and 4 juvenile hormone synthesis genes). For 26 randomly selected DEGs, real-time quantitative PCR (RT-qPCR) results showed that the relative expression levels of genes encoding several P450s, GSTs, heat shock protein (HSP) 68, vacuolar protein sorting-associated protein 13 (VPSAP13), sodium-coupled monocarboxylate transporter 1 (SCMT1), pupal cuticle protein (PCP), protein takeout (PT) and low density lipoprotein receptor adapter protein 1-B (LDLRAP1-B) were significantly up-regulated. Conversely, genes encoding esterase, sulfotransferase 1C4, proton-coupled folate transporter, chitinase 10, gelsolin-related protein of $125 \mathrm{kDa}$ (GRP), fibroin heavy chain (FHC), fatty acid synthase and some P450s were significantly down-regulated in response to fluralaner.

Conclusions: The transcriptome in this study provides more effective resources for the further study of $S$. litura whilst the DEGs identified sheds further light on the molecular response to fluralaner.

Keywords: Spodoptera litura, Fluralaner, Transcriptome, GABA receptor, Cytochrome P450 enzyme, Resistance, Agricultural pests

\footnotetext{
* Correspondence: zcq@njau.edu.cn; tanson_1@163.com

${ }^{1}$ Key Laboratory of Integrated Pest Management in Crops in Eastern China

(Ministry of Agriculture of China), College of Plant Protection, Nanjing

Agricultural University, Nanjing 210095, People's Republic of China

${ }^{2}$ Institute of Plant Protection, Hunan Academy of Agricultural Sciences,

Changsha 410125, People's Republic of China

Full list of author information is available at the end of the article
}

(c) The Author(s). 2020 Open Access This article is distributed under the terms of the Creative Commons Attribution 4.0 International License (http://creativecommons.org/licenses/by/4.0/), which permits unrestricted use, distribution, and reproduction in any medium, provided you give appropriate credit to the original author(s) and the source, provide a link to the Creative Commons license, and indicate if changes were made. The Creative Commons Public Domain Dedication waiver (http://creativecommons.org/publicdomain/zero/1.0/) applies to the data made available in this article, unless otherwise stated. 


\section{Background}

The common cutworm, Spodoptera litura Fabricius (Lepidoptera: Noctuidae), is a destructive polyphagous pest with a broad host plant range of more than 100 species of crops and vegetables [1]. It is widely distributed around the world and has evolved high resistance to most conventional insecticides, including carbamates, pyrethroids and organophosphates [2, 3]. As a result, farmers are using more insecticides leading to serious environmental pollution [4]. Therefore, exploring alternative and environmentallyfriendly insecticides is one important approach to reduce the economic loss from S. litura.

Fluralaner, as a novel isoxazoline insecticide, shows excellent insecticidal activity to agricultural pests including S. litura, the rice stem borer Chilo suppressalis Walker, the fall armyworm S. frugiperda Smith \& Abbot, the corn earworm Helicoverpa zea Boddie, the potato leafhopper Empoasca fabae (Harris), the western flower thrips Frankliniella occidentalis (Pergande) and the twospotted spider mite Tetranychus urticae Koch $[5,6]$. It exhibits no-cross resistance to the conventional GABAR targeting insecticides, such as phenylpyrazoles (e.g., fipronil) and cyclodienes (e.g., $\alpha$-endosulfan) [7]. Fluralaner was found to significantly reduce the weight of ticks on fluralaner-treated dogs [8]. Similarly, fluralaner postpones the development and reduces the fecundity of S. litura and significantly affects several detoxification-related genes [9]. However, the effect of fluralaner on S. litura at the transcriptomic level remains unclear.

The transcriptome has been widely used as a powerful tool for exploring the molecular mechanisms of genes that respond to insecticides and toxins. For instance, Cui et al. (2017) explored the responsive genes of the Asian corn borer (Ostrinia furnacalis Guenée) to flubendiamide [10]. Song et al. (2017) found that immunity-, metabolism- and Bt-related genes in S. litura midgut were responsive to Vip3Aa toxin, highlighting that trypsin was possibly involved in Vip3Aa activation [11]. $\mathrm{Xu}$ et al. (2014) found that in the carmine spider mite, T. cinnabarinus (Boisduval), 10 gene categories were putatively involved in insecticide resistance [12]. Marco et al. (2017) found that the defensome family genes of the mosquito, Anopheles stephensi Liston, responded to toxicants during insecticide exposure [13].

In the present study, the transcriptomes of control and fluralaner-treated $S$. litura were sequenced with the Illumina HiSeq Xten (IHX) platform (Illumina, Inc., San Diego, CA) and assembled according to the reference genome [14]. Hundreds of new genes were annotated by the NR, GO, COG and KEGG databases. The differentially expressed genes (DEGs) between control and fluralaner-treated $S$. litura were identified. In particular, detoxification enzyme genes and insecticide-targeted receptor genes were investigated, and fluralaner responsive genes relating to detoxification and development were also analyzed.

\section{Results \\ RNA-seq, sequence assembly, transcript analysis and functional classification}

The S. litura transcriptomic data were generated from 9 different libraries on the IHX platform. After removing the reads with adaptor and low quality, $70.97 \mathrm{~Gb}$ clean reads were produced with $\mathrm{Q}_{30} \geq 94.15 \%$. After alignment, clean reads $(85.71-88.22 \%)$ were successfully mapped to the $S$. litura genome, of which $85.83 \%$ mapped to exons and the others mapped to intergenic regions or introns. Finally, 16,926 genes were assembled in the S. litura transcriptome. Subsequently, 16,572 genes, including 451 new genes, were successfully annotated according to COG (6142), GO (7887), KEGG (6812), and NR (16,554) databases. In the transcriptome, 12,707 annotated genes were $\geq 1000 \mathrm{bp}$ long whilst 3850 annotated genes were between 300 and $999 \mathrm{bp}$.

Through comparing the $S$. litura transcripts against NR databases, most annotated genes ( $\geq$ 99.26\%) were highly homologous to Lepidopteran genes. To be specific, 15,884 (95.95\%) genes annotated by NR databases were homologous with those of S. litura, followed by 238 (1.44\%) and 147 (0.89\%) genes that were homologous to those of $H$. armigera Hübner and Heliothis virescens (Fabricius), respectively. Moreover, homologous genes identified by significant Blast hits from nine insect species are shown in Fig. 1a. According to the COG database, 6142 genes were annotated and classified into 25 specific categories and each gene was classified into at least one category (Fig. $1 \mathrm{~b}$ and Additional file 1). According to the GO database, 7887 annotated genes were primarily divided into three categories of "cellular component", "molecular function" and "biological process", and were further classified into 51 functional sub-categories, including 21, 16 and 14 sub-categories in the "biological process", "cellular component" and "molecular function" categories, respectively (Fig. 2 and Additional file 1). According to the KEGG database, 6812 annotated genes were involved in 154 pathways with "purine metabolism" (174 genes, 2.55\%), "carbon metabolism" (154 genes, 2.26\%) and "peroxisome" (143 genes, $2.10 \%$ being the largest three pathways (Additional file 2).

\section{Detoxification enzymes and insecticide-targeted genes}

Insecticide metabolism and resistance are mainly related to detoxification enzymes and insecticide-targeted genes of insects. In this study, the main detoxification enzyme genes (P450s, GSTs and CarEs) and insecticide-targeted genes (GABARs, GluCls, VGSCs, nAChRs, AChEs and RyRs) identified by BLAST searches against the NR 


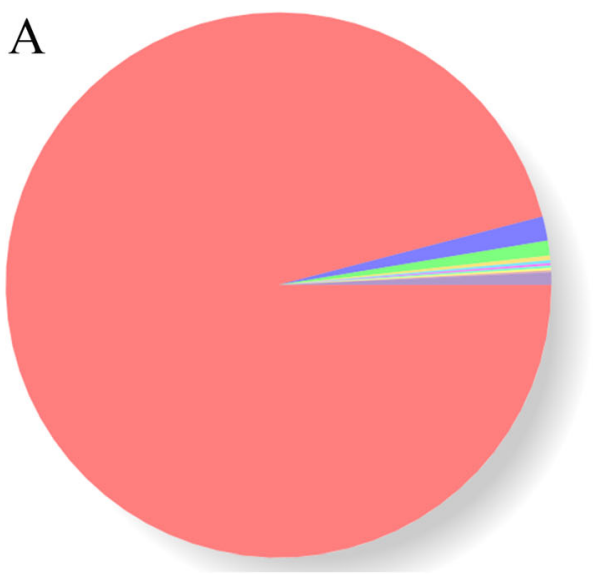

Spodoptera litura [15884, 95.95\%]

Helicoverpa armigera [238, 1.44\%]

Heliothis virescens [147, 0.89\%]

Bombyx mori [44, 0.27\%]

Amyelois transitella [29, 0.18\%]

Papilio machaon [24, 0.14\%]

Danaus plexippus [23, 0.14\%]

Papilio xuthus [22, 0.13\%]

Plutella xylostella $[20,0.12 \%]$

Others $[123,0.74 \%$ ]

B

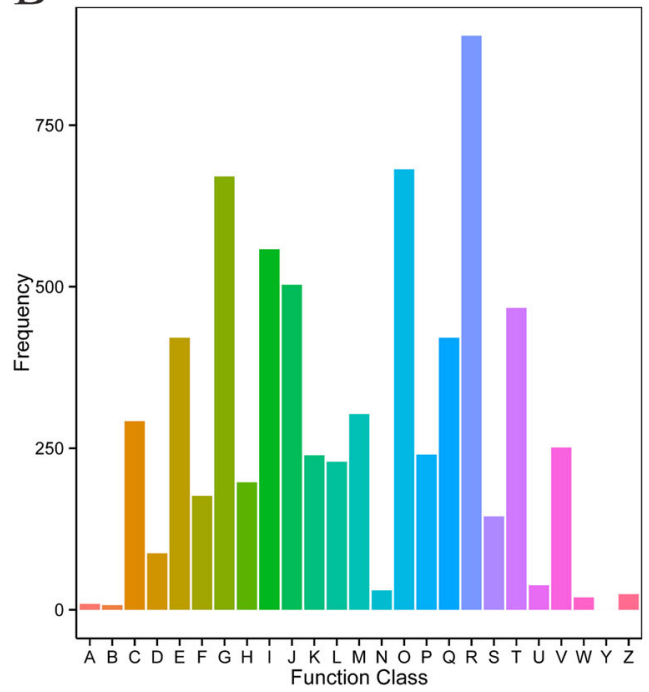

A: RNA processing and modification

B: Chromatin structure and dynamics

C: Energy production and conversion

D: Cell cycle control, cell division, chromosome partitioning

E: Amino acid transport and metabolism

F: Nucleotide transport and metabolism

G: Carbohydrate transport and metabolism

$\mathrm{H}$ : Coenzyme transport and metabolism

I: Lipid transport and metabolism

J: Translation, ribosomal structure and biogenesis

$\mathrm{J}$ : Translation, riboso
$\mathrm{K}$ : Transcription

L: Replication, recombination and repair

M: Cell wall/membrane/envelope biogenesis

$\mathrm{N}$ : Cell motility

O: Posttranslational modification, protein turnover, chaperones

$\mathrm{P}$ : Inorganic ion transport and metabolism

Q: Secondary metabolites biosynthesis, transport and catabolism

$\mathrm{R}$ : General function prediction only

$\mathrm{S}$ : Function unknown

T: Signal transduction mechanisms

U: Intracellular trafficking, secretion, and vesicular transport

$\checkmark$ : Defense mechanisms

W: Extracellular structures

Y: Nuclear structure

Z: Cytoskeleton

Fig. 1 Functional classification of annotated genes according to different database. Note: a species distribution of homology search of genes annotated by NR database; $\mathbf{b}$ distribution of COG functional classification of all annotated genes

database were analyzed. Compared with those from $B$. mori, Drosophila melanogaster Meigen, Apis mellifera Linnaeus, $H$. armigera, Plutella xylostella Linnaeus, 114 P450s genes were identified, 107 of which (Additional file 3) were clustered into CYP2, CYP3, CYP4 and mitochondrial (CYP $\mathrm{M})$ clades consisting of 44, 46, 10 and 7 genes, respectively (Additional file 4); 30 GSTs genes encoding proteins of 125-246 amino acid residues (Additional file 5) were identified and distributed into 6 clades: $\varepsilon(16), \sigma(5), \delta(4)$, microsomal (2), $\theta$ (1) and unclassified (2) clades (Additional file 6); 19 CarEs genes (Additional file 7) were identified (Additional file 8) as well. The identified insecticidetargeted genes included 6 putative ionotropic GABARs, 2 GluCls, 2 VGSCs, 15 nAChR subunits, 6 AChEs and 1 RyR that were annotated by the NR database (Additional file 9). However, phylogenetic analysis identified 5 ionotropic GABARs, $1 \mathrm{GluCl}, 12 \mathrm{nAChR}$ subunits and $2 \mathrm{AChE}$ genes (Additional file 10 and Additional file 11).
Expression profile analysis of fluralaner responsive genes In general, the transcriptome can represent the response of gene expression upon exposure to chemicals $[10,15]$. To determine the influence of fluralaner on the expression profile of genes, the change of genes was analyzed using the Fragments Per Kilobase of transcript per Million fragments mapped (FPKM) method [16]. A total of 3725 DEGs were found in $\mathrm{LC}_{30}$ and $\mathrm{LC}_{50}$ fluralaner-treated groups. The Venn diagram shows the number of shared and exclusive DEGs at each group, and a total of 3275 (1695 up-regulated and 1580 down-regulated) or 2491 (1500 up-regulated and 991 down-regulated) DEGs were found after exposure to $\mathrm{LC}_{30}$ and $\mathrm{LC}_{50}$ fluralaner compared with the control group, respectively. 100 DEGs were shared between the $\mathrm{LC}_{30}$ and $\mathrm{LC}_{50}$ treated groups (Fig. 3). 1209 and 406 DEGs were exclusive to $\mathrm{LC}_{30}$ and $\mathrm{LC}_{50}$ exposure to fluralaner, respectively, whilst 2041 genes DEGs were shared between all treated groups (Fig. 3). 

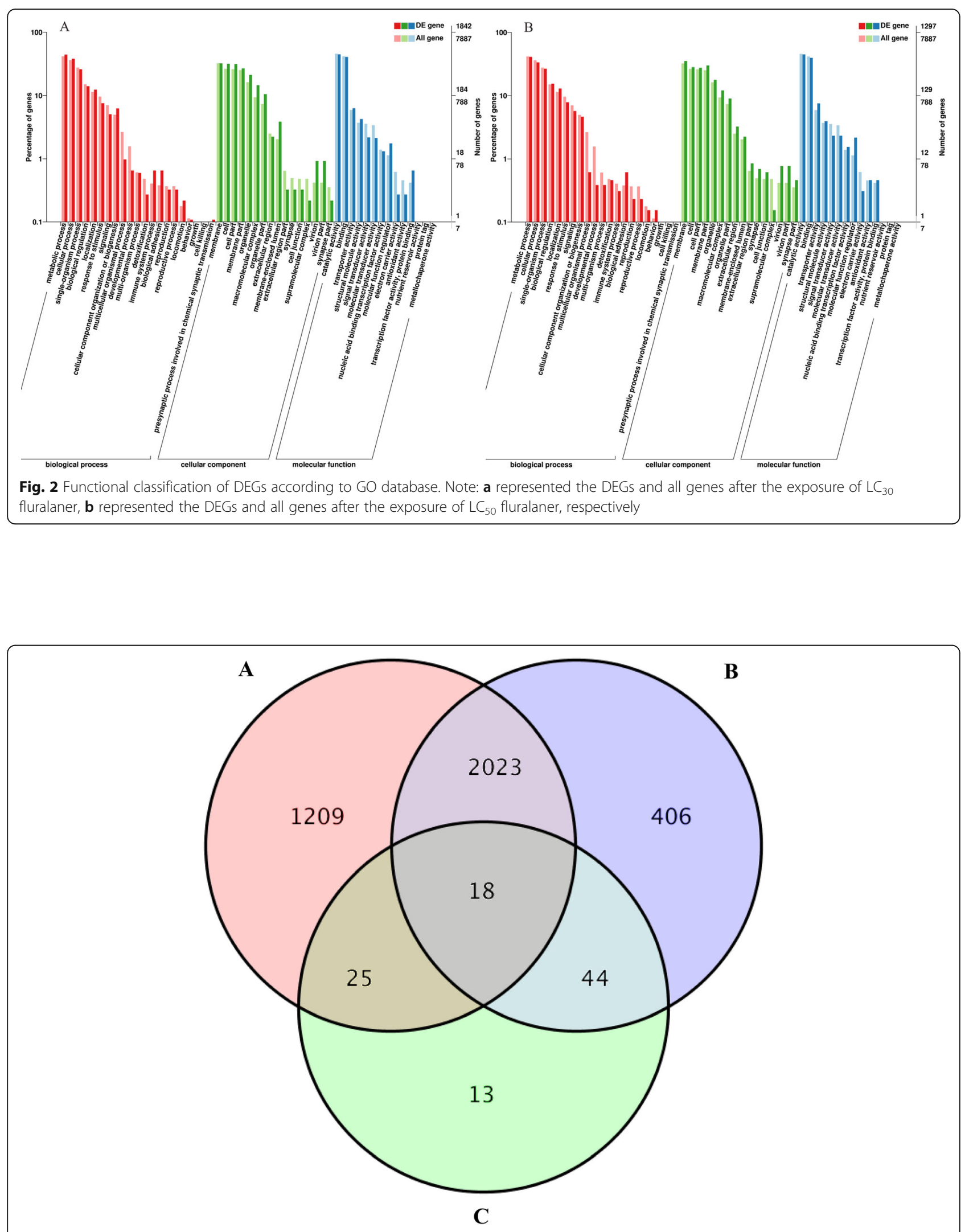

Fig. 3 Venn diagram of the DEGs among different treated groups. Note: a represented the DEGs between the control and $L C_{30}$ group; $\mathbf{b}$ represented the DEGs between the control and $L C_{50}$ group; $\mathbf{c}$ represented the $D E G s$ between the $L C_{30}$ and $L C_{50}$ group 
Subsequently, the DEGs in $\mathrm{LC}_{30}$ and $\mathrm{LC}_{50}$ fluralanertreated groups were analyzed by GO, COG and KEGG databases. 1842 and 1297 DEGs annotated by GO databases were divided into 50 and 48 sub-categories, respectively. The major sub-categories were "membrane" with 592 (32.14\%) and 455 (35.08\%) DEGs, respectively, "catalytic activity" with 823 (44.68\%) and 580 (44.72\%) DEGs, and "metabolic process" with 814 (44.19\%) and 531 (40.94\%) DEGs (Fig. 2). 1238 and 880 DEGs were annotated by the COG database and divided into 24 categories. The "general function prediction only" was the largest category with 187 (15.11\%) and 131 (14.89\%) DEGs, followed by $151(12.20 \%)$ and 128 (14.55\%) DEGs in the "posttranslational modification, protein turnover, chaperones" category (Additional file 12). 1728 and 1207 DEGs were annotated by the KEGG database and classified into 128 and 124 pathways with 62 and 41 DEGs being in the pathway "purine metabolism" in $\mathrm{LC}_{30}$ and $\mathrm{LC}_{50}$ fluralanertreated groups, respectively (Additional file 13).

As shown in Additional file 14, there were 43 and 30 DEGs of P450s after $\mathrm{LC}_{30}$ and $\mathrm{LC}_{50}$ exposure to fluralaner, respectively. Of these, 16 genes in both $\mathrm{LC}_{30}$ and $\mathrm{LC}_{50}$ treatment were overexpressed and annotated as CYP304A1 (gene9509), CYP49A1 (gene2558), CYP4C1like (gene3595, gene9175 and gene9174), CYP4D2-like (gene11881), CYP4G15-like (gene13430), CYP4V2-like (gene3598 and gene10859), CYP6A13 (gene9333), CYP6B7-like (gene4502), CYP9E2-like (gene17041, gene1218, gene12726 and gene15894) and CYP12A2like (gene11879). Six GSTs DEGs were found in both fluralaner-treated groups with only the "gene7753" (glutathione $S$-transferase 2-like) being significantly overexpressed. In addition, $3 \mathrm{CarE}$ genes were up-regulated after $\mathrm{LC}_{30}$ exposure, and were annotated as "venom CarE6-like" (gene8407), "CarE 1C-like" (gene5053) and "liver CarE B-1-like" (gene15885), which were not significantly changed after $\mathrm{LC}_{50}$ exposure. In exposure to both $\mathrm{LC}_{30}$ and $\mathrm{LC}_{50}$ fluralaner, a single chitin synthesis related gene annotated as "probable chitinase 10" (CHT10) (gene6005), and two juvenile hormone $(\mathrm{JH})$ synthesis related genes annotated as "JH esterase-like" (JHE-like) (gene1042), and "troso-like protein" (Tsl-like) (gene8419) were downregulated; the "JH acid $O$-methyltransferase-like" (Jhamtlike) (gene2891) and "Thamt-like isoform X2" (gene2887) genes were up-regulated.

\section{Verification of the DEGs by RT-qPCR assay}

In order to confirm the quality of the S. litura transcriptome and the results of DEGs, fourteen up-regulated genes, including four P450 genes (gene11879, gene1218, gene9333 and gene13430), three vacuolar protein sorting-associated protein 13 (VPSAP13) genes (gene5810, gene11398 and gene9409), two pupal cuticle protein (PCP) genes (gene13600 and gene13631), one heat shock protein (HSP) 68 (gene4060), sodium-coupled monocarboxylate transporter 1 (SCMT1) (gene6042), protein takeout (PT) (gene1073), low density lipoprotein receptor adapter protein 1-B (LDLRAP1-B) (gene8052) and GST (gene7753) gene, and 12 down-regulated genes, including 3 P450s genes (gene13788, gene10843 and gene5961), 2 esterase genes (gene11038 and gene1041), 2 sulfotransferase 1C4 genes (gene6203 and gene6209), and 1 protoncoupled folate transporter (gene334), chitinase 10 (gene6005), gelsolin-related protein of $125 \mathrm{kDa}$ (GRP) (gene4202), fibroin heavy chain (FHC) (gene7031) and fatty acid synthase (gene10548) gene, were selected for validation by RT-qPCR assay. Our results demonstrated that most of the selected up-regulated and down-regulated genes (71-92\%) showed the same expression trends, respectively, compared with the transcriptomic results (Fig. 4).

\section{Discussion}

In the present study, the transcriptome of S. litura untreated and treated with fluralaner was assembled based on the S. litura genomic database resulting in 16,572 genes being annotated according to NR, COG, GO, and KEGG databases. The number of annotated genes were

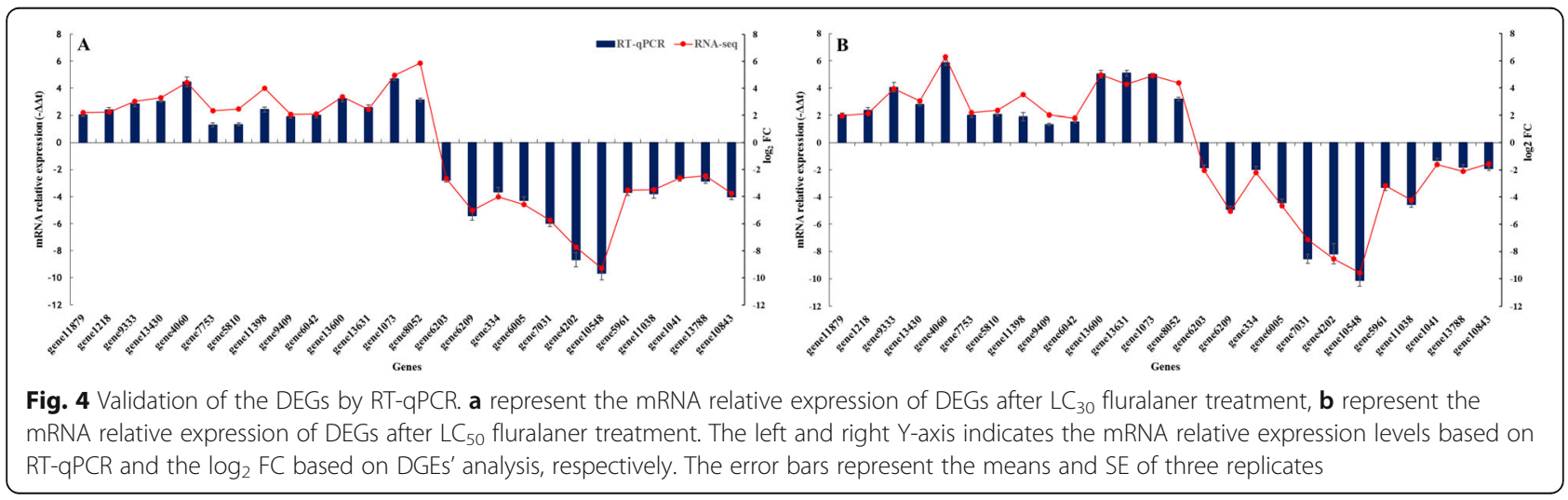


almost consistent to those $(16,161$ genes) in a de novo transcriptome of $S$.litura [17], whereas more than those (11,692 genes) based on sequence homology of $S$. litura [18] and the reference genome (15,317 genes) [14].

In insects, insecticides often induce the increase of metabolic activity of P450s, GSTs and CarEs [19, 20]. P450s are members of an important metabolic enzyme superfamily, which are involved in the metabolism of xenobiotic compounds such as insecticides and plant secondary metabolites [21]. Generally, there are four large clades of insect P450 genes that are CYP2, CYP3, CYP4 and CYPM [22]. In the present study, less genes of CYP3 and CYP4 clades and almost equal number of genes of CYP2 and CYPM clades were identified when compared to those from the reference genome $(61,58$, 11 and 8 genes of CYP3, CYP4, CYPM and CYP2 clades, respectively) [14]. The CYP3 and CYP4 are the largest clades and are mainly responsible for xenobiotic metabolism and insecticides resistance [22]. In this study, 90 genes accounting for $84.11 \%$ of the total $\mathrm{P} 450$ genes identified were clustered into the CYP3 and CYP4 clades, which were consistent with those containing 86.23\% P450 genes in the reference genome [14]. In this study, 16 P450 genes belonging to the CYP4, CYP6, CYP9 and CYP12 sub-families were overexpressed in fluralaner-treated groups (Additional file 14). Similarly, butene-fipronil, phoxim, cycloxaprid and chlorantraniliprole could significantly enhance the expression of CYP304A, CYP49A1, CYP4C1 and CYP9E2 in Drosophila melanogaster Meigen, B. mori, Periplaneta americana (Linnaeus) and Plutella xylostella Linnaeus, respectively [23-26]. Also, CYP4V2, CYP6A13-like and CYP12A2 were overexpressed in the insecticide resistant populations of Bemisia tabaci (Gennadius), Aphis glycines Matsumura and $M$. domestica, respectively [27-29]. Conversely, reduced CYP6B7 was found to increase larval susceptibility of $H$. armigera to fenvalerate [30]. Methanol was found to up-regulate the expression of CYP4D2 in D. melanogaster larvae [31] and CYP4G15 is probably important for the metabolism of endogenous compounds in the central nervous system of $D$. melanogaster [32]. We therefore speculate that up-regulating expression of $\mathrm{P} 450$ genes belonging to the CYP4, CYP6, CYP9 and CYP12 sub-families are likely involved in fluralaner metabolism in S. litura.

GSTs are a class of multifunctional detoxification enzymes that play an important role in the metabolism of insecticides [33]. The total number of GSTs genes identified in this study was less than those in the reference genome (47 GSTs) [14] but was more than those in $B$. mori (23 GSTs) (Additional file 6). In both fluralanertreated groups, six GST DEGs were detected and only "gene7753" belonging to the $\sigma$ clade was up-regulated, to 5.10- and 4.61-fold in $\mathrm{LC}_{30}$ and $\mathrm{LC}_{50}$ treatment, respectively. The $\sigma$ clade of GSTs play protective roles in processing xenobiotic compounds in insects [34, 35]. Similar to the $A$. aegypti GST-2 gene, which exhibited more than 8- fold mRNA levels in resistant strains than those in susceptible strains [36], the S. litura GST-2-like gene (gene7753) was over-expressed after exposure of fluralaner (Additional file 14) indicating that GST-2 may participate in metabolizing fluralaner. CarEs can hydrolyze a diverse range of carboxylates. In $T$. cinnabarinus, the expression levels of CarE-6 increased up to 1.64-fold under fenpropathrin-exposure [37] and the protein level of CarE1 was significantly increased in a chlorpyrifos-resistant strain of the small brown planthopper, Laodelphax striatellus Fallén [38]. Under $\mathrm{LC}_{30}$ fluralaner-treatment in S. litura, venom CarE-6-like (gene8407), CarE 1C-like (gene5053) and liver CarE B-1-like (gene15885) were up-regulated by 2.64-, 4.06- and 2.57- fold, respectively (Additional file 14), which indicates that these CarEs may be involved in the metabolism of fluralaner.

Fluralaner mainly acts on ionotropic GABARs [39, 40], therefore the identified ionotropic GABAR subunit genes would be useful for exploring the relationship between fluralaner and the S. litura GABAR. As shown in Additional file 10, three genes (gene14064, gene14068 and gene15324) were classified as resistance to dieldrin (RDL) subunits whilst the others were assigned to ligand-gated chloride channel homolog 3 (LCCH3) (gene11829), CG8916 (gene11828) and an unclassified clade (gene6139) (Additional file 10). However, it was notable that gene6139, annotated as a GABAR $\delta$ subunit, was possibly not a typical GABAR gene according to the phylogenetic tree analysis (Additional file 10). Similarly, "gene9312" was annotated as a GluCl subunit, "gene1980" and "gene2657" were annotated as nAChR subunits, and "gene555", "gene13723", "gene15777" and "gene4992" were annotated as AChE subunits in the NR database but not in the phylogenetic tree (Additional file 10 and Additional file 11). Therefore, we speculated that the phylogenetic analysis is very necessary while annotating the new genes. Notably, the 8916 subunit is considered as a GABAR-like gene in the phylogenetic tree analysis (Additional file 10), in agreement with a previous study in C. suppressalis [41], despite there still lacking functional evidence to date [42]. Previous studies have found that insecticide-treatment can affect expression of RDL in various insect species $[43,44]$. The over-expression of RDL1 (gene14064) and RDL2 (gene15324) after $\mathrm{LC}_{30}$ and $\mathrm{LC}_{50}$ exposure of fluralaner were similar to the relative mRNAs expression levels of GABAR from Leptinotarsa decemlineata (Say) treated with a sublethal concentration of fipronil [45]. $\mathrm{GluCl}$ mediates inhibitory synaptic transmission [46] and is identified as the secondary target of fluralaner [39]. Similar to many other species, such as D. melanogaster [47], Apis mellifera Linnaeus [46] and Tribolium 
castaneum (Herbst) [48], only one $\mathrm{GluCl}$ gene was identified in the $S$. litura transcriptome (Table 1 and Additional file 10).

Sublethal doses of fluralaner was found to reduce larval body weight, decrease pupation and emergence, and cause notched wings of adults in S. litura [9]. The DEGs of CHT 10, JHE, Tsl and Jhamt genes in the present study may mediate these effects in response to fluralaner exposure. The expression of CHT 10, JHE, Tsl and Jhamt were changed in the fluralaner-treated group (Additional file 14). As is known, CHT are important enzymes for chitin degradation and reconstruction, and play important roles in the shedding of the old cuticle and peritrophic matrix turnover in insects [49]. JHE is the primary $\mathrm{JH}$-specific degradation enzyme and indirectly regulates the $\mathrm{JH}$ titers [50]. Tsl is not just a specialized cue for Torso signaling but also acts independently in the control of body size and the timing of developmental progression [51]. Jhamt can transfer a methyl group from $S$-adenosyl-L-methionine to the carboxyl

Table 1 Putative insecticide-targeted genes identified in S. litura

\begin{tabular}{|c|c|c|c|c|}
\hline Insecticide class & Target & Gene name & $\begin{array}{l}\text { Gene } \\
\text { number }\end{array}$ & Length (bp) \\
\hline \multirow{6}{*}{$\begin{array}{l}\text { Cyclopentadienes; } \\
\text { Phenylpyrazole }\end{array}$} & \multirow[t]{5}{*}{ GABAR } & RDL1 subunit & gene14064 & 8257 \\
\hline & & RDL2 subunit & gene14068 & 7331 \\
\hline & & RDL3 subunit & gene15324 & 1633 \\
\hline & & 8916 subunit $^{a}$ & gene11828 & 1823 \\
\hline & & LCCH3 subunit & gene11829 & 1566 \\
\hline & GluCl & GluCl & gene3536 & 1645 \\
\hline \multirow[t]{2}{*}{ Pyrethroid } & \multirow[t]{2}{*}{ VGSC } & VGSC & gene491 & 9853 \\
\hline & & VGSC & gene9046 & 6872 \\
\hline \multirow{13}{*}{$\begin{array}{l}\text { Neonicotinoids; } \\
\text { Sarcotoxin }\end{array}$} & \multirow[t]{13}{*}{$\mathrm{nAChR}$} & a1 subunit & gene8842 & 5075 \\
\hline & & a2 subunit & gene8844 & 3665 \\
\hline & & a3 subunit & gene9676 & 2010 \\
\hline & & a4 subunit & gene9375 & 4199 \\
\hline & & a5 subunit & gene7363 & 2055 \\
\hline & & a6 subunit & gene7613 & 3853 \\
\hline & & a7 subunit & gene7328 & 3269 \\
\hline & & a8 subunit & gene8875 & 1809 \\
\hline & & a9 subunit & gene863 & 1414 \\
\hline & & $\beta 1$ subunit & gene7327 & 2158 \\
\hline & & $\beta$ subunit & gene8228 & 1792 \\
\hline & & $\beta$ subunit & gene1694 & 1307 \\
\hline & & $\beta$ subunit & gene8227 & 2050 \\
\hline \multirow[t]{2}{*}{ Organophosphorous } & \multirow[t]{2}{*}{ AChE } & AChE1 & gene1175 & 2608 \\
\hline & & AChE2 & gene9347 & 5592 \\
\hline Diamides & RyR & RyR & gene11613 & 16,054 \\
\hline
\end{tabular}

Note: ${ }^{a}$ the 8916 gene was considered as a GABAR-like subunit in Drosophila [47] group of $\mathrm{JH}$ acids to produce active $\mathrm{JHs}$ in the corpora allata in insects (Jhamt in D. melanogaster). In line with the present study, knockdown of CHT10 induced lethal phenotypes, developmental arrest and high mortality in Nilaparvata lugens (Stål) [49]. Also, depletion of B. mori JHE by CRISPR/Cas9 resulted in the extension of larval stages [50] and Tsl mutation could lead to developmental delay in D. melanogaster [51]. Furthermore, overexpression of Jhamt resulted in a pharate adult lethal phenotype in D. melangaster [52]. Therefore, the transcriptional change of these related genes may contribute to the abnormal growth and development of S. litura.

\section{Conclusions}

In conclusion, transcriptome analysis of S. litura based on the reference genome was provided in the present study. The identified DEGs may help uncover the possible molecular mechanisms underlying responses to fluralaner in S. litura. In particular, P450s may be involved in the detoxification of fluralaner in vivo. This study, therefore, may facilitate the identification of genes involved in fluralaner resistance and may offer useful information for exploring novel insecticides to control S. litura.

\section{Methods}

\section{Insects and chemicals}

A laboratory strain of S. litura was reared as described in our previous report [9]. Fluralaner (a.i. $\geq 99 \%$ ) was purified from Bravecto ${ }^{\circ}$ (Merck \& Co. Inc., Isando, South Africa) as described in Sheng et al. (2019) [53]. The 3rd instar larvae of S. litura were chosen for the experiment and three treatments (45 larvae per treatment) were used in the present study. Larvae in the control group were fed with artificial food containing acetone and in the fluralaner-treated groups with artificial food containing different sublethal concentrations $\left[\mathrm{LC}_{30}\right.$ $\left(0.59 \mathrm{mg}\right.$ of fluralaner per $\mathrm{kg}$ of artificial food, $\left.\mathrm{mg} \cdot \mathrm{kg}^{-1}\right)$ and $\mathrm{LC}_{50}\left(0.78 \mathrm{mg} \cdot \mathrm{kg}^{-1}\right)$, respectively] of fluralaner dissolved in acetone. After $24 \mathrm{~h}, 15$ alive and normal $S$. litura from each treatment were randomly collected, equally divided into three $1.5 \mathrm{~mL}$ Eppendorf tubes, respectively, immediately frozen in liquid nitrogen and stored at $-80^{\circ} \mathrm{C}$ until use.

\section{Total RNA extraction, cDNA library construction and IHX sequencing}

Total RNA was isolated from whole body of S. litura from each group (the control and fluralaner-treated groups) by TRIzol ${ }^{\mathrm{mt}}$ Reagent (Invitrogen, Carlsbad, CA). The concentration and integrity of total RNA were measured using NanoDrop 2000 (Thermo Fisher Scientific, Waltham, MA) and with the RNA Nano 6000 Assay Kit by Agilent Bioanalyzer 2100 system (Agilent Technologies, Santa Clara, CA), respectively. 
RNA sequencing libraries were generated from each sample and the sequencing was carried out by BioMarker (Beijing, China) following the manual of the NEB Next ${ }^{\circ}$ Ultra $^{\text {Tw }}$ RNA Library Prep Kit (NEB, Ipswich, MA) in Illumina. Briefly, $1 \mu \mathrm{g}$ of total RNA from each sample was enriched using magnetic beads with Oligo (dT) and broken into short fragments, which was carried out using divalent cations under elevated temperature by adding NEB Next First Strand Synthesis Reaction Buffer (5x) (NEB). These short fragments of mRNA served as templates for synthesis of the first-strand complementary DNA (cDNA) with random hexamer primers and Moloney Murine Leukemia Virus (M-MuLV) Reverse Transcriptase (NEB). Using buffer, dNTPs, RNase $\mathrm{H}$ and DNA Polymerase I, the second-strand cDNA was subsequently synthesized. Remaining overhangs were converted into blunt ends via exonuclease/ polymerase activities. After adenylation of 3' ends of DNA fragments, NEB Next Adaptor with hairpin loop were ligated for hybridization. The purified cDNAs were subjected to end repair by adding an adenosine triphosphate (A) base to the $3^{\prime}$ end and connected with sequencing adaptor. Suitable fragments of cDNAs were extracted by the AMPure XP system (Beckman Coulter, Beverly, CA). Three microliter of USER Enzyme (NEB) was used for size selection, and adaptor-ligated cDNAs at $37^{\circ} \mathrm{C}$ for $15 \mathrm{~min}$ followed by $5 \mathrm{~min}$ at $95^{\circ} \mathrm{C}$ before PCR. Then PCR was performed with Phusion High Fidelity DNA polymerase (NEB), Universal PCR primers, and Index (X) Primer (NEB). PCR products were purified with the AMPure XP system (Beckman Coulter) and library quality was assessed on the Agilent Bioanalyzer 2100 system (Agilent).

The clustering of the index coded samples was carried out on a cBot Cluster Generation System (Illumina) using TruSeq PE Cluster Kit v4-cBot-HS (Illumina) according to the manufacturer's instruction. After cluster generation, the prepared libraries were sequenced on an Illumina platform and paired end reads were generated.

\section{Mapping and annotation of the clean reads}

Raw reads in the fastq format were first processed by inhouse perl scripts. Subsequently, clean reads were obtained from raw reads by removing reads with low quality, adaptor and poly-N. The intergenic regions and introns were identified by comparing the reads to the general feature format (GFF) file of the reference genome [14]. The clean reads with a perfect match or with only one mismatch were mapped to the reference genome of S. litura [54] by the Hisat2 software [55]. The mapped reads were assembled into the transcriptome by the StringTie method [56] and all the genes were functionally annotated and classified by Blast [57] according to the databases of Clusters of Orthologous Groups (COG) [58], Gene Ontology (GO) [59], Kyoto Encyclopedia of Genes and Genomes
(KEGG) [60] and Non-redundant protein (NR) [61]. In addition, new genes from the unannotated sequences encoding more than 50 amino acids were identified and analyzed by Blast using the above mentioned databases.

\section{Identification of detoxification enzymes and insecticide- targeted genes}

Genes encoding detoxification enzymes including P450s, GSTs, CarEs, and insecticide-targeted genes including ionotropic GABARs, GluCl, VGSCs, nAChRs, RyR and AChEs, were identified from the $S$. litura transcriptome. Referring to the previous studies [10, 21, 62], all of the confirmed protein sequences were aligned with $S$. litura, B. mori, D. melanogaster and A. mellifera, then the phylogenetic trees were mapped with 1000 bootstrap replications using the neighbor-joining method to evaluate the branch strength of each tree using MEGA 7 [63]. The phylogenetic trees were further annotated via the EvolView online tool [64]. Referring to previous publications $[46,48,65]$, the phylogenetic tree of cys-loop ligand-gated ion channel proteins was constructed using Clustal X [66] and TreeView software [67].

\section{Identification and validation of DEGs}

The relative expression levels of genes among the control, $\mathrm{LC}_{30^{-}}$and $\mathrm{LC}_{50}$-treated groups were analyzed using DEGseq method [68], which provided statistical routines for determining differential expression in digital gene expression data using a model based on the negative binomial distribution. The fold change $(F C \geq 2)$ and false discovery rate $(\mathrm{FDR}<0.01)$ from corrected $P$-value were used as a judgment standard for the significant differences in gene expression between two samples [15]. The DEGs were annotated, classified and analyzed by databases of COG, GO, KEGG, and NR. The RTqPCR assay was performed to validate the randomly selected 26 DEGs using TB Green ${ }^{\mathrm{Tm}}$ Premix Ex Taq II (Tli RNaseH Plus) (Takara Biotechnology (Dalian) Co., Ltd., Liaoning, China) on a Quant Studio ${ }^{\mathrm{Tm}} 6$ and 7 Flex Real-Time PCR System (Life Technologies Corporation, Carlsbad, CA) according to the procedures from Liu et al. (2018) [9]. The housekeeping gene of EF-1 $\alpha$ was selected as an internal standard to eliminate variations in mRNA and cDNA quantity and quality [69]. Three technical replications per biological replicate were conducted and the relative mRNA expression levels of genes were analyzed using the $2^{-\Delta \Delta \mathrm{Ct}}$ method [70]. The primers used in this study are listed in Additional file 15. All values were expressed as means \pm standard error (SE) and the statistical figures were constructed by Microsoft Excel (Microsoft, Redmond, WA). 


\section{Supplementary information}

Supplementary information accompanies this paper at https://doi.org/10. 1186/s12864-020-6533-0

\author{
Additional file 1: Text S1. Gene annotation with COG and GO \\ databases. \\ Additional file 2. All pathways annotated by KEGG database. \\ Additional file 3. Cytochrome P450 nucleotide sequences of the $S$. \\ litura transcriptome.
}

Additional file 4. Neighbor-joining phylogenetic analysis of the P450s genes from D. melanogaster, H. armigera, P. xylostella, S. litura and B. mori.

Additional file 5. Glutathione S-transferase nucleotide sequences of the S. litura transcriptome.

Additional file 6. Neighbor-joining phylogenetic analysis of the GST genes from D. melanogaster, A. mellifera, S. litura and B. mori.

Additional file 7. Carboxylesterase nucleotide sequence of the $S$. litura transcriptome

Additional file 8. Neighbor-joining phylogenetic analysis of the CarE genes from D. melanogaster, H. armigera, P. xylostella, S. litura and B. mori.

Additional file 9. Nucleotide sequences of annotated insecticidetargeted genes of the S. litura transcriptome.

Additional file 10. Neighbor-joining phylogenetic analysis of the cys-loop ligand-gated ion channel superfamily genes from S. litura and other species. The subunits sequence used are as follow: Am-alpha1 (AAY87890.1), Am-alpha2 (AAS48080.1), Am-alpha3 (AAY87891.1), Am-alpha4 (AAY87892.1), Am-alpha5 (AJE70263.1), Am-alpha6 (AAY87894.1), Am-alpha7 (AAR92109.1), Am-alpha8 (NP_001011575.1), Am-alpha9 (AAY87896.1), Am-beta1 (AAY87897.1), Am-beta2 (AAY87898.1), Am-GluCl (ABG75737.1), Am-RDL (AJE68941.1), Am-GRD (AJE68942.1), Am-LCCH3 (AJE68943.1), Am-CG8916 (NP_001071290.1), Am-HisCl (ABG75739.1), Am-pHCl (ABG75741.1), Am-CG6927 (ABG75747.1), Am-CG12344 (ABG75746.1), Dm-alpha1 (AGB96296.1), Dm-alpha2 (NP 524482.1), Dm-alpha3 (NP 525079.3), Dm-alpha4 (NP_001097669.2), Dm-alpha5 (NP_995708.1), Dm-alpha6 (NP_995674.1), Dm-alpha7 (NP 996514.1), Dm-beta1 (NP 523927.2), Dm-beta2 (NP_524483.1), Dm-beta3 (AAF51485.1), Dm-RDL (NP_523991.2), Dm-LCCH3 (NP 996469.1), Dm-GRD (CAA55144.1), Dm-CG8916 (AAF48539.4), Dm-HisCl1 (AAL74413.1), Dm-HisCl2 (AAL74414.1), Dm-NtR (NP 651958.2), Dm-pHCl1 (NP_001034025.2), Dm-pHCl2 (NP_651861.1), Dm-CG6927 (AAF45992.1), Dm-CG7589 (AAF49337.2), Dm-CG12344 (NP 610619.2), Bm-alpha1 (XP 021203289.1), Bm-alpha2 (NP_001103397.1), Bm-alpha3 (NP_001103387.2), Bm-alpha4 (NP_001166816.1), Bm-alpha5 (NP_001166811.1), Bm-alpha6 (NP 001166813.1), Bm-alpha7 (NP 001166818.1), Bm-alpha8 (NP_001166817.1), Bm-alpha9 (ABV72691.1), Bm-beta1 (ABV72692.1), Bm-beta2 (ABV72693.1), Bm-beta3 (ABV45510.1), Bm-RDL1 (ADM88014.1), Bm-RDL2 (NP_001182629.1), Bm-RDL3 (NP_001182630.1), Bm-LCCH3 (BAT57341.1), Bm-GluCl (BAO58781.1), Bm-pHCl (BAX77827.1).

Additional file 11. Phylogenetic analysis of the AChE genes. Additional file 12. Functional classification of DEGs according to COG database. Note: A represented the DEGs and all genes after the exposure of $L C_{30}$ fluralaner, $B$ represented the DEGs and all genes after the exposure of $L C_{50}$ fluralaner, respectively.

Additional file 13. Functional classification of DEGs according to KEGG database.

Additional file 14. Changes of DEGs related to detoxification and development of S. litura after exposure of fluralaner.

Additional file 15. Primers for RT-qPCR validation.

\section{Abbreviations}

AChE: Acetyl cholinesterase; bp: Base pair; CarE: Carboxylesterase; cDNA: Complementary DNA; CHT10: Chitinase 10; COG: Clusters of Orthologous Groups; DEG: Differentially expressed gene; GABAR: $\gamma^{-}$ aminobutyric acid receptor; GluCl: Glutamate-gated chloride channel; GO: Gene Ontology; GRP: Gelsolin-related protein of 125 kDa; HSP: Heat shock protein; JH: Juvenile hormone; Jhamt: Juvenile hormone acid Omethyltransferase; JHE: Juvenile hormone esterase; KEGG: Kyoto Encyclopedia of Genes and Genomes; LC 50 : Median lethal concentration; LDLRAP1-B: Low density lipoprotein receptor adapter protein 1-B; nAChR: Nicotinic acetylcholine receptor; NR: Non-redundant; PCP: Pupal cuticle protein; PT: Protein takeout; RT-qPCR: Real-time quantitative PCR; RyR: Ryanodine receptor; SCMT1: Sodium-coupled monocarboxylate transporter; Tsl: Trosolike protein; VGSC: Voltage-gated sodium channels; VPSAP13: Vacuolar protein sorting-associated protein 13

\section{Acknowledgments}

We give special thanks to Dr. Andrew K. Jones (Oxford Brookes University) for improvement of English language throughout the manuscript.

\section{Authors' contributions}

CQZ designed the experiments; ZQJ and DL performed the experiments; ZQJ, $\Pi$ and YCP analyzed the data; CQZ, $\Pi$, and ZJH wrote and revised the manuscript. All authors reviewed the manuscript. All authors read and approved the final manuscript.

\section{Funding}

This work was supported by the National Key R\&D Program of China [grant number 2016YFD0300700/2016YFD0300706]; Postgraduate Research \&Practice Innovation Program of Jiangsu Province [grant number KYCX19_0530]; and Chinese Universities Scientific Fund [grant number KYZ201710]; the National Natural Science Foundation of China [grant number 31830075]. The funding bodies played no role in the design of the study and collection, analysis, and interpretation of data and in writing the manuscript.

\section{Availability of data and materials}

All data are presented within the article and the additional supporting files.

\section{Ethics approval and consent to participate}

Not applicable.

\section{Consent for publication}

Not applicable.

\section{Competing interests}

The authors declare that they have no competing interests.

\section{Author details}

${ }^{1}$ Key Laboratory of Integrated Pest Management in Crops in Eastern China (Ministry of Agriculture of China), College of Plant Protection, Nanjing Agricultural University, Nanjing 210095, People's Republic of China. ${ }^{2}$ Institute of Plant Protection, Hunan Academy of Agricultural Sciences, Changsha 410125, People's Republic of China. ${ }^{3}$ Present address: Institute of Entomology, Jiangxi Agricultural University, Nanchang 330045, China.

Received: 30 April 2019 Accepted: 23 January 2020

Published online: 03 February 2020

\section{References}

1. Ahmad M, Saleem MA, Sayyed AH. Efficacy of insecticide mixtures against pyrethroid- and organophosphate-resistant populations of Spodoptera litura (Lepidoptera: Noctuidae). Pest Manag Sci. 2009;65:266-74.

2. Tong H, Su Q, Zhou X, Bai L. Field resistance of Spodoptera litura (Lepidoptera: Noctuidae) to organophosphates, pyrethroids, carbamates and four newer chemistry insecticides in Hunan, China. J Pestic Sci. 2013:86:599-609.

3. Shad SA, Sayyed AH, Fazal S, Saleem MA, Zaka SM, Ali M. Field evolved resistance to carbamates, organophosphates, pyrethroids, and new chemistry insecticides in Spodoptera litura fab. (Lepidoptera: Noctuidae). J Pestic Sci. 2012;85:153-62.

4. Casida JE, Quistad GB. Golden age of insecticide research: past, present, or future? Annu Rev Entomol. 1998:43:1-16.

5. Lahm GP, Daniel C, Barry JD, Pahutski TF, Smith BK, Long JK, Benner EA, Holyoke CW, Kathleen J, Ming X. 4-Azolylphenyl isoxazoline insecticides acting at the GABA gated chloride channel. Bioorg Med Chem Lett. 2013;23: 3001-6. 
6. Sheng CW, Jia ZQ, Wu HZ, Luo XM, Liu D, Song PP, Xu L, Peng YC, Han ZJ, Zhao CQ. Insecticidal spectrum of fluralaner to agricultural and sanitary pests. J Asia-Pacif Entomol. 2017;20:1213-8.

7. Gassel M, Wolf C, Noack S, Williams H, llg T. The novel isoxazoline ectoparasiticide fluralaner: selective inhibition of arthropod $\gamma$-aminobutyric acid- and L-glutamate-gated chloride channels and insecticidal/acaricidal activity. Insect Biochem Mol Biol. 2014;45:111-24.

8. Williams H, Demeler J, Taenzler J, Roepke RKA, Zschiesche E, Heckeroth AR. A quantitative evaluation of the extent of fluralaner uptake by ticks (Ixodes ricinus, Ixodes scapularis) in fluralaner (Bravecto ${ }^{\mathrm{TM} M}$ ) treated vs. untreated dogs using the parameters tick weight and coxal index. Parasit Vectors. 2015;8:352.

9. Liu D, Jia Z-Q, Peng Y-C, Sheng C-W, Tang T, Xu L, Han Z-J, Zhao C-Q. Toxicity and sublethal effects of fluralaner on Spodoptera litura Fabricius (Lepidoptera: Noctuidae). Pestic Biochem Physiol. 2018:52:8-16.

10. Cui L, Rui C, Yang D, Wang Z, Yuan H. De novo transcriptome and expression profile analyses of the Asian corn borer (Ostrinia furnacalis) reveals relevant flubendiamide response genes. BMC Genomics. 2017;18:20.

11. Song F, Chen C, Wu S, Shao E, Li M, Guan X, Huang Z. Transcriptional profiling analysis of Spodoptera litura larvae challenged with Vip3Aa toxin and possible involvement of trypsin in the toxin activation. Sci Rep. 2016;6:23861.

12. Xu Z, Zhu W, Liu Y, Liu X, Chen Q, Peng M, Wang X, Shen G, He L. Analysis of insecticide resistance-related genes of the carmine spider mite Tetranychus cinnabarinus based on a de novo assembled transcriptome. PLoS One. 2014:9:e94779.

13. De Marco L, Sassera D, Epis S, Mastrantonio V, Ferrari M, Ricci I, Comandatore F, Bandi C, Porretta D, Urbanelli S. The choreography of the chemical defensome response to insecticide stress: insights into the Anopheles stephensi transcriptome using RNA-Seq. Sci Rep. 2017;7:41312.

14. Cheng T, Wu J, Wu Y, Chilukuri RV, Huang L, Yamamoto K, Feng L, Li W, Chen Z, Guo H, Liu J, Li S, Wang X, Peng L, Liu D, Guo Y, Fu B, Li Z, Liu C, Chen Y, Tomar A, Hilliou F, Montagné N, Jacquin-Joly E, d'Alençon E, Seth RK, Bhatnagar RK, Jouraku A, Shiotsuki T, Kadono-Okuda K, Promboon A, Smagghe G, Arunkumar KP, Kishino H, Goldsmith MR, Feng Q, Xia Q, Mita K. Genomic adaptation to polyphagy and insecticides in a major east Asian noctuid pest. Nat Ecol Evol. 2017;1:1747-56.

15. Shi T-F, Wang Y-F, Liu F, Qi L, Yu L-S. Sublethal effects of the neonicotinoid insecticide thiamethoxam on the transcriptome of the honey bees (Hymenoptera: Apidae). J Econ Entomol. 2017;110:2283-9.

16. Trapnell C, Williams BA, Pertea G, Mortazavi A, Kwan G, van Baren MJ, Salzberg SL, Wold BJ, Pachter L. Transcript assembly and quantification by RNA-Seq reveals unannotated transcripts and isoform switching during cell differentiation. Nat Biotechnol. 2010;28:511-5.

17. Gong L, Wang H, Qi J, Han L, Hu M, Jurat-Fuentes JL. Homologs to cry toxin receptor genes in a de novo transcriptome and their altered expression in resistant Spodoptera litura larvae. J Invertebr Pathol. 2015;129:1-6.

18. Zhang Y-N, Zhu X-Y, Fang L-P, He P, Wang Z-Q, Chen G, Sun L, Ye Z-F, Deng D-G, Li J-B. Identification and expression profiles of sex pheromone biosynthesis and transport related genes in Spodoptera litura. PLoS One. 2015;10:e0140019.

19. Ranson H, Claudianos C, Ortelli F, Abgrall C, Hemingway J, Sharakhova MV, Unger MF, Collins FH, Feyereisen R. Evolution of supergene families associated with insecticide resistance. Science. 2002;298:179.

20. Oakeshott JG, Devonshire AL, Claudianos C, Sutherland TD, Horne I, Campbell PM, Ollis DL, Russell RJ. Comparing the organophosphorus and carbamate insecticide resistance mutations in cholin- and carboxylesterases. Chem Biol Interact. 2005;157-158:269-75.

21. Zimmer CT, Maiwald F, Schorn C, Bass C, Ott M-C, Nauen R. A de novo transcriptome of European pollen beetle populations and its analysis, with special reference to insecticide action and resistance. Insect Mol Biol. 2014;23:511-26.

22. Feyereisen R. Evolution of insect P450. Biochem Soc Trans. 2006;34:1252-5.

23. Arain MS, Shakeel M, Elzaki MEA, Faroog M, Hafeez M, Shahid MR, Shah SAH, Khan FZA, Shakeel Q, Salim AMA, Li G-Q. Association of detoxification enzymes with butene-fipronil in larvae and adults of Drosophila melanogaster. Environ Sci Pollut Res. 2018;25:10006-13.

24. Li F, Ni M, Zhang H, Wang B, Xu K, Tian J, Hu J, Shen W, Li B. Expression profile analysis of silkworm P450 family genes after phoxim induction. Pestic Biochem Physiol. 2015;122:103-9.

25. Zhang J, Zhang Y, Li J, Liu M, Liu Z. Midgut transcriptome of the cockroach Periplaneta americana and its microbiota: digestion, detoxification and oxidative stress response. PLoS One. 2016;11:e0155254.
26. Gao Y, Kim K, Kwon DH, Jeong IH, Clark JM, Lee SH. Transcriptome-based identification and characterization of genes commonly responding to five different insecticides in the diamondback moth, Plutella xylostella. Pestic Biochem Physiol. 2018;144:1-9.

27. Yang X, Xie W, Wang S, Wu Q, Xu B, Zhou X, Zhang Y. Resistance status of the whitefly, Bemisia tabaci, in Beijing, Shandong and Hunan areas and relative levels of CYP4V2 and CYP6CX1 mRNA expression. Plant Prot. 2014; 40:70-5.

28. Xi J, Pan Y, Bi R, Gao X, Chen X, Peng T, Zhang M, Zhang H, Hu X, Shang Q. Elevated expression of esterase and cytochrome P450 are related with lambda-cyhalothrin resistance and lead to cross resistance in Aphis glycines Matsumura. Pestic Biochem Physiol. 2015;118:77-81.

29. Guzov VM, Unnithan GC, Chernogolov AA, Feyereisen R. CYP12A1, a mitochondrial cytochrome P450 from the house fly. Arch Biochem Biophys. 1998;359:231-40.

30. Tang T, Zhao C, Feng X, Liu X, Qiu L. Knockdown of several components of cytochrome P450 enzyme systems by RNA interference enhances the susceptibility of Helicoverpa armigera to fenvalerate. Pest Manag Sci. 2012; 68:1501-11.

31. Wang SP, He GL, Chen RR, Li F, Li GQ. The involvement of cytochrome P450 monooxygenases in methanol elimination in Drosophila melanogaster larvae. Arch Insect Biochem Physiol. 2012;79:264-75.

32. Maibèche-Coisne M, Monti-Dedieu L, Aragon S, Dauphin-Villemant C. A new cytochrome P450 from Drosophila melanogaster, CYP4G15, expressed in the nervous system. Biochem Biophys Res Commun. 2000;273:1132-7.

33. Bass $C$, Field LM. Gene amplification and insecticide resistance. Pest Manag Sci. 2011;67:886-90.

34. Mittapalli O, Neal JJ, Shukle RH. Tissue and life stage specificity of glutathione S-transferase expression in the hessian fly, Mayetiola destructor: implications for resistance to host allelochemicals. J Insect Sci. 2007;7:20.

35. Yamamoto K, Fujii H, Aso Y, Banno Y, Koga K. Expression and characterization of a sigma-class glutathione $\mathrm{S}$-transferase of the fall webworm, Hyphantria cunea. Biosci Biotechnol Biochem. 2007;71:553-60.

36. Grant DF, Matsumura F. Glutathione S-transferase 1 and 2 in susceptible and insecticide resistant Aedes aegypti. Pestic Biochem Physiol. 1989;33:132-43.

37. Wei P, Shi L, Shen G, Xu Z, Liu J, Pan Y, He L. Characteristics of carboxylesterase genes and their expression-level between acaricidesusceptible and resistant Tetranychus cinnabarinus (Boisduval). Pestic Biochem Physiol. 2016;131:87-95.

38. Zhang $Y, L i ~ S, X u L, H f G, Z i$ J, Wang L, He P, Fang J. Overexpression of carboxylesterase-1 and mutation (F439H) of acetylcholinesterase-1 are associated with chlorpyrifos resistance in Laodelphax striatellus. Pestic Biochem Physiol. 2013;106:8-13.

39. Ozoe $Y$, Asahi M, Ozoe F, Nakahira K, Mita T. The antiparasitic isoxazoline A1443 is a potent blocker of insect ligand-gated chloride channels. Biochem Biophys Res Commun. 2010;391:744-9.

40. Casida JE, Durkin KA. Neuroactive insecticides: targets, selectivity, resistance, and secondary effects. Annu Rev Entomol. 2013;58:99-117.

41. Jia Z-Q, Sheng C-W, Tang T, Liu D, Leviticus K, Zhao C-Q, Chang X-L. Identification of the ionotropic GABA receptor-like subunits from the striped stem borer, Chilo suppressalis Walker (Lepidoptera: Pyralidae). Pestic Biochem Physiol. 2019;155:36-44.

42. Wei Q, Wu SF, Gao CF. Molecular characterization and expression pattern of three GABA receptor-like subunits in the small brown planthopper Laodelphax striatellus (Hemiptera: Delphacidae). Pestic Biochem Physiol. 2017;136:34-40.

43. Xie L, Li S, Jiang W. Cloning and expression profile of nicotinic acetylcholine receptor a5 subunit gene from Leptinotarsa decemlineata. J Nanjing Agric Univ. 2018:41:293-301

44. Yu X, Wang M, Kang M, Liu L, Guo X, Xu B. Molecular cloning and characterization of two nicotinic acetylcholine receptor $\beta$ subunit genes from Apis cerana cerana. Arch Insect Biochem Physiol. 2011;77:163-78.

45. Fu J. Identification and characterization of aminobutyric acid receptor genes in Leptinotarsa decemlineata. Nanjing: Nanjing Agricultural University; 2016.

46. Jones AK, Sattelle DB. The cys-loop ligand-gated ion channel superfamily of the honeybee, Apis mellifera. Invert Neurosci. 2006;6:123-32.

47. Knipple DC, Soderlund DM. The ligand-gated chloride channel gene family of Drosophila melanogaster. Pestic Biochem Physiol. 2010;97:140-8.

48. Jones AK, Sattelle DB. The cys-loop ligand-gated ion channel gene superfamily of the red flour beetle, Tribolium castaneum. BMC Genomics. 2007;8:327. 
49. $X i$ Y, Pan P-L, Ye Y-X, Yu B, Xu H-J, Zhang C-X. Chitinase-like gene family in the brown planthopper, Nilaparvata lugens. Insect Mol Biol. 2015;24:29-40.

50. Zhang Z, Liu X, Shiotsuki T, Wang Z, Xu X, Huang Y, Li M, Li K, Tan A. Depletion of juvenile hormone esterase extends larval growth in Bombyx mori. Insect Biochem Mol Biol. 2017;81:72-9.

51. Johnson TK, Crossman T, Foote KA, Henstridge MA, Saligari MJ, Forbes Beadle L, Herr A, Whisstock JC, Warr CG. Torso-like functions independently of torso to regulate Drosophila growth and developmental timing. Proc Natl Acad Sci U S A. 2013;110:14688-92.

52. Niwa R, Niimi T, Honda N, Yoshiyama M, Itoyama K, Kataoka H, Shinoda T. Juvenile hormone acid O-methyltransferase in Drosophila melanogaster. Insect Biochem Mol Biol. 2008;38:714-20.

53. Sheng C-W, Huang Q-T, Liu G-Y, Ren X-X, Jiang J, Jia Z-Q, Han Z-J, Zhao CQ. Neurotoxicity and mode of action of fluralaner on honeybee Apis mellifera L. Pest Manag Sci. 2019:75:2901-9.

54. Southwest University. Genome information for Spodoptera litura. 2017: https://www.ncbi.nlm.nih.gov/assembly/GCF_002706865.1/.

55. Kim D, Langmead B, Salzberg SL. HISAT: a fast spliced aligner with low memory requirements. Nat Methods. 2015;12:357-60.

56. Pertea M, Pertea GM, Antonescu CM, Chang T-C, Mendell JT, Salzberg SL. StringTie enables improved reconstruction of a transcriptome from RNA-seq reads. Nat Biotechnol. 2015:33:290-5.

57. Altschul SF, Madden TL, Schäffer AA, Zhang J, Zhang Z, Miller W, Lipman DJ. Gapped BLAST and PSI-BLAST: a new generation of protein database search programs. Nucleic Acids Res. 1997;25:3389-402.

58. Tatusov RL, Galperin MY, Natale DA, Koonin EV. The COG database: a tool for genome-scale analysis of protein functions and evolution. Nucleic Acids Res. 2000;28:33-6.

59. Ashburner M, Ball CA, Blake JA, Botstein D, Butler H, Cherry JM, Davis AP, Dolinski K, Dwight SS, Eppig JT, Harris MA, Hill DP, Issel-Tarver L, Kasarskis A, Lewis S, Matese JC, Richardson JE, Ringwald M, Rubin GM, Sherlock G. Gene ontology: tool for the unification of biology. Nat Genet. 2000;25:D158-D69.

60. Kanehisa M, Goto S, Kawashima S, Okuno Y, Hattori M. The KEGG resource for deciphering the genome. Nucleic Acids Res. 2004;32:D277-D80.

61. Deng Y, Li J, Wu S, Zhu Y, Chen Y, He F. Integrated nr database in protein annotation system and its localization. Comput Eng. 2006;32:71-9.

62. Xu G, Wu S-F, Wu Y-S, Gu G-X, Fang Q, Ye G-Y. De novo assembly and characterization of central nervous system transcriptome reveals neurotransmitter signaling systems in the rice striped stem borer, Chilo suppressalis. BMC Genomics. 2015;16:525.

63. Kumar S, Stecher G, Tamura K. MEGA7: molecular evolutionary genetics analysis version 7.0 for bigger datasets. Mol Biol Evol. 2016;33:1870-4.

64. He Z, Zhang H, Gao S, Lercher MJ, Chen W-H, Hu S. Evolview v2: an online visualization and management tool for customized and annotated phylogenetic trees. Nucleic Acids Res. 2016;44:W236-W41.

65. Jones AK, Bera AN, Lees K, Sattelle DB. The cys-loop ligand-gated ion channel gene superfamily of the parasitoid wasp, Nasonia vitripennis. Heredity. 2010;104:247.

66. Gibson TJ, Higgins DG, Plewniak F, Thompson JD, Jeanmougin F. The CLUSTAL_X windows interface: flexible strategies for multiple sequence alignment aided by quality analysis tools. Nucleic Acids Res. 1997;25:4876-82.

67. Page RDM. TREEVIEW : an application to display phylogenetic trees on personal computers. Comput Appl Biosci. 1996;12:357-9.

68. Wang $L$, Feng Z, Wang $X$, Wang $X$, Zhang $X$. DEGseq: an R package for identifying differentially expressed genes from RNA-seq data. Bioinformatics. 2009;26:136-8

69. Huang S-J, Qin W-J, Chen Q. Cloning and mRNA expression levels of cytochrome P450 genes CYP4M14 and CYP4S9 in the common cutworm Spodoptera litura (Fabricius). Sci Agric Sin. 2010:43:3115-24

70. Livak KJ, Schmittgen TD. Analysis of relative gene expression data using real-time quantitative PCR and the $2^{-\Delta \Delta C T}$ method. Methods. 2001;25:402-8.

\section{Publisher's Note}

Springer Nature remains neutral with regard to jurisdictional claims in published maps and institutional affiliations.

\section{Ready to submit your research? Choose BMC and benefit from:}

- fast, convenient online submission

- thorough peer review by experienced researchers in your field

- rapid publication on acceptance

- support for research data, including large and complex data types

- gold Open Access which fosters wider collaboration and increased citations

- maximum visibility for your research: over $100 \mathrm{M}$ website views per year

At BMC, research is always in progress.

Learn more biomedcentral.com/submissions 